Canadian Oncology

Nursing Journal

Revue canadienne

de soins infirmiers

en oncologie

Volume 27, Issue 2 • Spring 2017

elSSN: 2368-8076 


\section{Les bienfaits de l'écriture : étude qualitative portant sur les ateliers communautaires destinés aux survivantes du cancer du sein}

\section{par Roanne Thomas, Wendy Gifford et Chad Hammond}

\section{RÉSUMÉ}

Survivre au cancer du sein peut s'avérer difficile vu le peu de soutien communautaire disponible dans ce domaine. En ce sens, lécriture constitue une intervention psychosociale prometteuse, mais la plupart des études n'ont évalué cette activité que du point de vue individuel. Pour faire contrepoint, notre étude qualitative $(n=12)$ a analysé l'expérience vécue par des femmes ayant participé à un atelier d'écriture communautaire. L'analyse des entrevues effectuées dans le cadre de l'étude, ainsi que des carnets d'écriture tenus par les participantes et des enregistrements de l'atelier a mis en évidence trois thèmes : a) avoir un endroit rassurant pour se confier; b) se donner le droit de penser à soi et chercher à retrouver son équilibre; c) surmonter ses craintes et combattre l'incertitude. Ces thèmes illustrent les besoins non comblés liés à l'impact émotionnel du cancer du sein, ainsi que le pouvoir de l'écriture en groupe en vue de favoriser le bien-être. Les programmes (communautaires) peuvent pallier aux lacunes présentes dans les soins offerts aux survivants du cancer, en créant des endroits rassurants pour l'expression des émotions, tout en soutenant les participantes dans la rédaction de nouveaux récits axés sur le bien-être et la tenue d'un journal.

\section{INTRODUCTION}

Le taux de survie des patientes atteintes de cancer du sein pour les cinq dernières années est de $88 \%$ au Canada (Statistiques canadiennes sur le cancer, 2015). S'il est vrai que ce taux fait état de progrès médicaux considérables, il s'accompagne néanmoins de difficultés pour les survivantes. Ainsi, des besoins en soins psychosociaux et en réadaptation liés aux effets secondaires tels que la douleur constante ou un lymphœdème peuvent surgir. La période de transition à la suite

\section{AU SUJET DES AUTEURS}

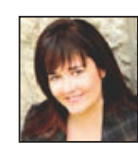

Auteure-ressource : Roanne Thomas, Ph.D., Professeure titulaire, École des sciences de la réadaptation, Faculté des sciences de la santé, Université d'Ottawa, 451 Smyth Road (salle 3068, RGN), Ottawa, ON, K1H 8M5, Tél. : 613-562-5800, poste 8645, Téléc. : 613-562-5428; courriel : roanne.thomas@uottawa.ca

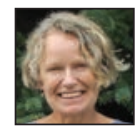

Wendy Gifford, Ph.D., Professeure agrégée, École des sciences infirmières, Faculté des sciences de la santé, Université d'Ottawa, 451 Smyth Road (salle 1118, RGN), Ottawa, ON, K1H 8M5; courriel : wgifford@uottawa.ca

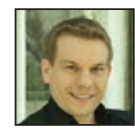

Chad Hammond, Ph.D., Boursier postdoctoral, École des sciences de la réadaptation, Faculté des sciences de la santé, Université d'Ottawa, 451 Smyth Road (salle 1125, RGN), Ottawa, ON, K1H 8M5; courriel : chammond@uottawa.ca

https://doi.org/10.5737/23688076272186195 des traitements comprend également des changements de rôles au sein de la famille, dans le milieu de travail et dans la communauté (Mackenzie, 2014; Pelusi, 2001). Il faut savoir négocier ce genre de changement et retrouver un juste équilibre tout en investissant les efforts requis pour conserver un bien-être physique et psychosocial. Tous ces domaines ont été mis en lumière dans les documents importants de survie (Howell et al, 2009; The Institute of Medicine et Conseil national de recherches, 2005) et sont présentés dans les récentes conceptualisations de la détresse (Bultz et Johansen, 2011; Holland et Bultz, 2007; Waller, Groff, Hagen, Bultz et Carlson, 2012) avec la nécessité des soins axés sur la personne. La période qui suit le traitement est maintenant largement reconnue en tant que transition essentielle chez les femmes tandis qu'elles terminent le traitement et retrouvent une vie satisfaisante (Fitch et al, 2009).

Une recommandation clé en matière de prestations de services de survie (Howell, Hack, Oliver, Chulak, Mayo, Aubin et Tompson, 2011) est d'offrir du soutien au moment de la transition vers la survie, que ce soit au moyen de sensibilisation, de programmes communautaires ou de réadaptation. Malgré tout, on constate dans les lignes directrices de pratique pancanadienne destinées aux adultes que selon toute vraisemblance, les besoins psychosociaux et en soins de soutien ne sont pas bien satisfaits. (Howell et al, 2009; Howell, Hack, Oliver, Chulak, Mayo, Aubin, Chasen et al, 2011). Thompson et collègues (2014) en fournissent un exemple concret dans leur étude sur les soins après traitement lorsqu'ils disent que seulement $49 \%$ des survivantes du cancer du sein qui ont reçu leur diagnostic de un à cinq ans plus tôt ont eu l'impression que leurs besoins psychologiques et spirituels ont été comblés après le traitement. Une autre étude ayant examiné le cas de 1323 adultes survivants du cancer a indiqué que plus de $50 \%$ d'entre eux manquaient de soutien pendant les six mois suivant le diagnostic, et que $37 \%$ avaient des besoins non comblés de niveau « modéré à élevé » en lien avec la peur, la fatigue, la détresse et l'incertitude, de même que le travail et la sexualité (Boyes, Girgis, D’Este et Zucca, 2012). Même si l'on reconnaît la détresse comme le « sixième signe vital » dans les soins contre le cancer, les survivants semblent avoir un accès limité aux interventions psychosociales (Bultz et Johansen, 2011). À long terme, les soins aux survivantes du cancer devraient couvrir diverses sphères - individuelle, familiale, communautaire - parce que la survie est un processus dynamique qui continue toute la vie (Pelusi, 2001). Même si les soins de soutien se terminent souvent avec la fin des soins actifs, les inquiétudes des survivants du cancer vont 
bien au-delà de cette période (Thomas-MacLean, 2004a). Les interventions communautaires axées sur la création, comme l'écriture, peuvent être adaptées aux besoins des participants et résoudre certaines difficultés liées à la survie.

De plus en plus de chercheurs constatent que le travail créatif est significatif sur le plan personnel et contribue à la santé et au bien-être (Camic, 2008; Cox et al, 2010; Sonke, Rollins, Brandman et Graham-Pole, 2009; Stuckey et Nobel, 2010). En plus de l'intérêt croissant pour l'art-thérapie, notre conscience du potentiel que présentent la recherche et l'application des connaissances axées sur les arts s'aiguise (Barone et Eisner, 2012; Knowles et Cole, 2008; Rieger et Schultz, 2014); celles-ci alimentent désormais les stratégies de survie au cancer. Cependant, l'exploration systématique de l'efficacité d'une vaste gamme de pratiques créatives axées sur les arts, comme les arts visuels et l'écriture, est relativement récente et sous-explorée (Rieger et Schultz, 2014).

En général, il est bien reconnu que l'engagement dans une démarche créative peut améliorer l'état émotif ainsi que la santé dans son ensemble (Stuckey et Nobel, 2010). Dans une recension des recherches en art-thérapie, Stuckey et Nobel (2010) citent plusieurs essais cliniques aléatoires où, par rapport aux groupes témoins, les groupes d'intervention ont montré une amélioration des résultats pouvant être associée aux arts (y compris l'écriture). Ces études comprenaient des participants atteints de maladies chroniques, comme le VIH et la fibromyalgie; leurs résultats dénotaient diverses améliorations : diminution de la détresse, de la douleur, de la fatigue et de l'utilisation des soins de santé. Les activités créatives (par exemple l'écriture et les arts visuels comme la peinture) sont associées à une diminution des symptômes de dépression, de stress et de fatigue, tout en améliorant le bien-être chez les patients atteints de cancer (Haltiwanger, Rojo et Funk, 2011; Visser et Op'T Hoog, 2008). Une revue systématique d'essais cliniques aléatoires et de différentes activités d'art-thérapie ayant porté sur 1576 patients cancéreux (total de 27 études) a révélé une diminution de l'anxiété, de la dépression et de la douleur, ainsi qu'une amélioration moyenne à élevée de la qualité de vie (Puetz, Morley et Herring, 2013). Même si cette revue de littérature démontre l'efficacité d'une approche axée sur les arts, la plupart des devis de recherche utilisés dans ces études n'ont utilisé qu'une seule intervention, soit la musicothérapie. L'utilisation de l'écriture est donc un domaine d'intervention ayant été peu évalué dans les études répertoriées.

Une autre limite de ces études est que la plupart d'entre elles sont des interventions ayant été effectuées uniquement en milieu hospitalier (Stuckey et Nobel, 2010). Les auteurs ont remarqué que les interventions de type communautaire sont fréquemment négligées et recommandent que de futures recherches soient effectuées au sein des groupes communautaires. Les interventions de groupe intégrant la pratique créative ont permis d'atténuer la détresse et de favoriser l'adaptation et la santé mentale (Monti et al, 2006; Rosedale, 2009; Visser et Op'T Hoog, 2008). Par exemple, une étude en milieu communautaire avec des survivantes du cancer du sein a relevé des améliorations dans de multiples sphères, y compris une amélioration de la qualité de vie, de même qu'une réduction de la fatigue et du stress post-traumatique (Lu, Zheng, Young, Kagawa-Singer et Loh, 2012). La recherche à ce sujet indique également que les activités de groupe peuvent aider les participants à se prendre en charge, à augmenter leur capacité d'adaptation, ainsi qu'à améliorer l'humeur et la qualité de vie (Haltiwanger et al, 2011; Luzzatto et Gabriel, 2000).

Une approche particulière de l'écriture, qui a été largement étudiée et permet d'améliorer l'équilibre émotionnel, est « l'écriture d'expression » proposée à l'origine par Pennebaker. Une revue systématique récente de la littérature sur l'écriture d'expression chez les patients atteints de cancer (1986-2012) a notamment relevé 13 études utilisant un devis expérimental (Merz, Fox et Malcarne, 2014). Ces études présentaient des résultats prometteurs, notamment des effets bénéfiques de l'écriture sur le sommeil, la douleur et les autres symptômes psychologiques et physiques. Cependant, plusieurs limites des études sur les effets bénéfiques de l'écriture d'expression ont été soulignées et liées aux variables inhérentes à ces études. Premièrement, Merz et collègues (2014) soulignent que l'écriture d'expression est effectuée individuellement, avec peu d'instructions, et que les résultats liés à certains indicateurs mesurés suggèrent que le nombre habituel de séances prévues (de 1 à 4) serait insuffisant (Merz et al., 2014). Pareillement, Nicholls (2009) soutient que l'écriture expressive et les résultats associés sont limités parce que l'intervention se restreint à quelques courtes séances d'écriture individuelles avec peu d'instructions pour orienter les participants (Nicholls, 2009). Deuxièmement, aucune des études répertoriées ne présente d'analyse qualitative détaillée des écrits produits par les participants. Merz et collègues (2014) avancent qu'une analyse qualitative de ces récits pourrait procurer des données qui éclaireraient les mécanismes d'écriture efficaces et les personnes susceptibles d'en profiter. Ces données pourraient aussi servir de base à l'élaboration d'une étude axée sur l'intervention. En outre, des données qualitatives pourraient aider à mieux comprendre les effets - généralement difficiles à mesurer - de l'écriture expressive sur la croissance personnelle et la capacité à trouver un sens aux événements de la vie, ce qui favorise le bien-être psychosocial (Merz et al, 2014). Troisièmement, seulement une étude permettait aux participants d'adapter l'intervention à leurs besoins (c'est-àdire de choisir librement sur quoi ils écriraient). Cette étude de Jensen-Johansen et collèques (2012) est celle qui a affiché les résultats les plus concluants parmi les 13 répertoriées et analysées, indiquant qu'une intervention axée sur la liberté d'écriture pourrait être la plus efficace (Merz et al, 2014). Finalement, certains auteurs ont mentionné que l'écriture expressive n'était pas toujours bien ancrée dans la «vraie vie » (Merz et al, 2014). Le cas échéant, des travaux de recherche ont montré qu'écrire avec un groupe de soutien ciblé, animé par un mentor d'expérience, pouvait procurer des bienfaits à ceux qui n'avaient pas tiré avantage de l'approche axée sur l'écriture expressive individuelle (Nicholls, 2009). L'approche préconisée par Nicholls est de plus en plus populaire compte tenu de sa combinaison d'activités effectuées et analysées individuellement et en groupe. 
L'intervention communautaire que nous avons conçue tenait compte de quelques-unes des limites relevées dans les travaux de recherche antérieurs; elle prévoyait ainsi des activités individuelles et de groupe, avait des lignes directrices souples et adaptables, proposait des exercices de lecture et de réflexion. Les participantes avaient notamment l'occasion de prendre du recul sur le premier jet et d'effectuer des tâches par étape (exploration du point de vue adopté et autres révisions, par exemple). L'intervention sur l'écriture a été faite conjointement et a inclus le soutien d'un rédacteur professionnel. Elle a ainsi pu s'appuyer sur les bienfaits démontrés de l'écriture tout en dépassant les limites relevées pour l'écriture d'expression et dans les études associées.

Notre étude avait pour but d'évaluer une intervention fondée sur l'écriture expressive avec des survivantes du cancer $\mathrm{du}$ sein afin d'explorer qualitativement les effets possibles du programme sur le bien-être des participantes. L'un des buts secondaires était par ailleurs de documenter sur le plan qualitatif la transition vécue lors de la phase de survie, les expériences vécues et relatées dans les écrits des participantes, ainsi que l'utilisation de différentes méthodes d'intervention. Puisque ces objectifs concordent entre eux, il est difficile de discuter isolément de ceux-ci. Le présent article met l'accent sur l'expérience vécue par les femmes lors de l'utilisation de l'écriture expressive, de même que lors de leur participation aux ateliers proposés.

\section{MÉTHODOLOGIE}

Afin d'explorer les effets bénéfiques éventuels de l'intervention et de documenter la transition et les difficultés qui y sont associées, nous avons opté pour un protocole de recherche qualitatif s'appuyant sur la décision interprétative (Thorne, 2008). Des données qualitatives ont été recueillies par l'analyse de carnets d'écriture, de projets visuels (ici, la production de collages des participantes photographiés ensuite), ainsi que de sondages et entrevues téléphoniques avec les participantes. Le verbatim des ateliers d'écriture a aussi été enregistré et transcrit intégralement afin de réaliser subséquemment, une analyse en profondeur.

Nos travaux de recherche et notre intervention se sont fondés sur de solides cadres théoriques élaborés dans le contexte des connaissances entourant les récits de maladie (Bury, 1982; Frank, 1995; Malchiodi, 2013; Sinding et Wiernikowski, 2008; Thomas-MacLean, 2004b). En résumé, malgré le fait que le mécanisme précis lié aux effets bénéfiques de l'écriture et d'autres pratiques d'art-thérapie sur la santé reste inconnu, les spécialistes affirment que l'expérience de la maladie vient perturber le récit de vie des participants. La détresse (émotionnelle) vécue durant cette période peut être atténuée en utilisant des procédés qui favorisent la réflexion sur le sens de la vie dans des cadres précis (Malchiodi, 2013). Par exemple, les écrits qui dominent culturellement au niveau de l'expérience de la maladie ne donnent pas la possibilité aux femmes de questionner les idéologies reliées à la survie, qui sont purement positives. Celles-ci peuvent donc ressentir l'étiquette de « survivante » comme étant limitative, puisqu'elle n'encourage pas ces femmes à s'exprimer sur la peur ou la douleur qu'elles ressentent (Malchiodi, 2013; Thomas-MacLean, 2004b). La production d'écrits réflexifs aux côtés de pairs ayant eu un vécu similaire peut créer un endroit où il est rassurant d'exprimer ses émotions et d'adapter ses récits en fonction de la maladie (Collie, Bottorff et Long, 2006).

Notre intervention axée sur l'écriture a été offerte sous forme de deux ateliers intensifs d'une durée totale de 14 heures (deux journées de 7 heures). Les deux ateliers ont été espacés de deux semaines pour donner du temps d'écriture individuelle. Le contenu, les outils et les méthodes d'enseignement de l'atelier ont tous été élaborés en collaboration avec un rédacteur professionnel dont l'approche incorporait une orientation holistique de l'acte d'écrire, ture; par exemple, les participantes étaient invitées à effectuer un exercice de pleine conscience qui incluait la respiration profonde et une réflexion sur leur soi incarné (Thomas-MacLean, 2005) avant le début des exercices d'écriture. Les participantes ont également reçu un carnet et un guide d'écriture.

\section{Participantes et paramètres}

Après avoir reçu l'approbation du comité d'éthique de recherche de notre université, nous avons commencé le recrutement des participantes par une en annonce de notre étude à l'intérieur d'un centre communautaire pour les survivants au cancer situé à Ottawa, en Ontario (le site qui a accueilli les ateliers). Douze femmes ayant reçu un diagnostic de cancer du sein entre 6 mois et 9 ans plus tôt ( 2 ans en moyenne), ont participé aux ateliers offerts. Elles étaient âgées entre 43 et 75 ans, avec une moyenne d'âge de 54 ans. Parmi les 12 participantes, 8 d'entre elles ont mentionné avoir déjà discuté de leur cancer du sein avec un groupe de soutien ou un professionnel de la santé. Les patientes ont effectué entre 6 et 17 entrées dans leur carnet d'écriture (moyenne de 11 entrées) et ont produit entre 13 à 41 pages au total (moyenne de 23 pages). Parmi ces 12 participantes, 11 ont effectué et partagé des collages.

\section{Analyse}

Toutes les discussions des ateliers ont été enregistrées, y compris les discussions de groupe et en petits groupes. Les transcriptions mot à mot des enregistrements ont été vérifiées par un assistant de recherche. Les entrevues téléphoniques servaient à revoir les réponses écrites des participantes à des sondages utilisant des questions ouvertes sur les ateliers; elles ont uniquement servi à étoffer celles émanant des sondages, et n'ont donc pas été enregistrées. Les notes des entrevues ont été analysées par triangulation, à partir des questions de sondage correspondantes. Les collages des participantes ont été photographiés, et les entrées dans les carnets d'écriture ont été numérisées puis sauvegardées en format PDF. Toutes les données ont ensuite été transférées pour analyse dans NVivo 10. Les transcriptions, les entrées des carnets d'écriture, les réponses aux sondages et les notes d'entrevue ont été lues globalement, puis ligne par ligne afin d'en extraire les passages importants des entrevues, en suivant les lignes directrices établies pour valider la recherche descriptive interprétative (Thorne, 2008). Les passages sélectionnés ont servi à produire des codes initiaux qui ont servi de base pour tout schématiser. Les catégories thématiques qui en ont découlé ont ensuite été 
rassemblées afin d'illustrer les principales difficultés associées à la transition vers la survie et à l'intervention. Le codage initial a été effectué par l'auteur principal, puis révisé et analysé plus en profondeur à l'aide de discussions avec les deuxième et troisième auteures.

La crédibilité et la schématisation (Leininger, 1994; Munhall et Oiler-Boyd, 1993) ont fréquemment été utilisées comme critères pour évaluer la qualité d'une analyse. Ceux-ci ont été établis par comparaison de sources de données multiples. Par exemple, les effets bénéfiques du groupe étaient mis en évidence dans les récits écrits et la transcription des ateliers. Autre exemple, les entrevues individuelles de suivi ont permis de clarifier les données écrites provenant de l'évaluation des ateliers. Finalement, toutes les participantes ont été invitées à partager lors d'une séance où les résultats préliminaires de l'étude leur ont été communiqués. Cinq d'entre elles y ont participé. On leur a ensuite demandé si les résultats correspondaient à leur expérience quant au programme. Elles ont toutes répondu par l'affirmative et ont confirmé la nécessité d'avoir des programmes de soutien aux survivants centrés sur une activité telle que l'activité d'écriture, contrairement à une simple discussion tenue dans les groupes de soutien traditionnel.

\section{RÉSULTATS}

Trois principaux thèmes sont ressortis de l'analyse des carnets d'écriture, des sondages sur les ateliers, des entrevues, ainsi que du verbatim des ateliers. Étant donné que l'analyse des collages dépasse la portée du présent manuscrit, elle sera présentée dans une publication future. Ces trois thèmes sont : a) avoir un endroit rassurant pour se confier; b) se donner le droit de penser à soi et chercher à retrouver son équilibre; c) surmonter ses craintes et combattre l'incertitude. Le premier thème est associé à l'expression des émotions (colère, détresse...) perçues comme étant négatives. En dehors du groupe, les participantes avaient l'impression qu'elles ne pouvaient pas partager véritablement ces émotions avec d'autres personnes. Le deuxième thème illustre comment les exigences des rôles joués sont perçues par ces femmes et occasionnent, en contexte de maladie, des situations qui les ont amenées à revoir l'équilibre entre les différents domaines de leur vie quotidienne et à s'accorder la permission de réfléchir sur les impacts du cancer. Le troisième thème porte sur l'expérience vécue par participantes, sur l'incertitude liée au cancer, ainsi que sur leur désir de trouver des manières d'y faire face. Ensemble, les thèmes qui ont émergé indiquent le besoin d'interventions communautaires de nature participative pour aider les femmes atteintes de cancer du sein à bien vivre les transitions et à bien gérer leur détresse.

\section{Avoir un endroit rassurant pour se confier}

Nous entendons ici la notion « d'endroit » dans son sens large : elle ne désigne pas seulement le lieu physique où se déroulent les ateliers, mais s'étend jusqu'au matériel utilisé pour la libre expression (comme les carnets d'écriture), ainsi qu'au contexte social des ateliers. Létablissement de ces divers endroits a commencé lors de la première séance et s'est poursuivi tout au long de l'expérience. Comme la création de ces endroits rassurants sert de base au processus complet, de l'écriture jusqu'à l'édition et au partage des textes, ces espaces sont fondamentaux à tous les thèmes; ainsi, leur ordre de présentation n'est pas entièrement chronologique.

Dès le début des ateliers, des endroits rassurants ont été créés de plusieurs façons. De manière inattendue, certaines participantes ont en fait rapidement assumé la création d'un milieu confortable. Par exemple, pendant les présentations initiales, une participante ${ }^{1}$ a mentionné ceci : « Je suis vraiment très heureuse d'être ici aujourd'hui et d'avoir la possibilité de rencontrer d'autres femmes. » Elle a exprimé à quel point il était utile d'écouter les témoignages des autres femmes. Ensuite, un dialogue entre une participante et l'un des animateurs qui s'est aussi produit dans la première demi-heure du premier atelier a aussi renforcé l'établissement d'un espace favorisant la confidence :

Participante: J'aimerais dire que durant mon parcours avec le cancer, beaucoup de choses positives me sont arrivées, mais il $y$ a aussi eu du négatif. J'aimerais pouvoir parler des choses négatives et positives. Je ne voudrais pas repartir d'ici en ayant seulement surfé sur le sujet, parce que ça n'a pas tout le temps été super au cours de la dernière année. Et si j'ai envie de sortir du négatif, je veux me sentir à l'aise de le faire.

Animateur: Parfait. Je suis content que vous en parliez parce qu'il ne faut surtout pas éviter de parler des choses négatives. Nous ne sommes pas au Club optimiste. Vous n'avez pas à transformer les choses pour leur donner une image positive. Cet atelier porte sur vos écrits, bien à vous.

Dans l'exemple ci-dessus, la création de l'espace rassurant s'est faite à deux : la participante, d'abord, qui a fait part de ses besoins, puis l'animateur, dans sa réponse à cette intervention.

Le processus s'est poursuivi de manière continue, ce qui s'est reflété dans les commentaires d'une participante vers la fin de la première journée, qui a dit ceci après le partage des écrits :

On met ici en mots des choses que j'ai ressenties, mais que je ne suis pas arrivée à exprimer. C'est cette expérience commune, je crois, qui permet de se dire «Oui, voilà! C'est comme ça que je me suis sentie. »C'est arriver à ça, je pense, qui donne toute sa valeur au fait d'écrire, au fait de lire.

Une autre participante qui revenait sur la première journée a expliqué :

On doit être capable de discuter de choses qui sont là, mais dont on n'a pas réellement conscience. L'autre chose est juste d'écouter ce que les autres ont à dire. Ça me surprend de pouvoir partager de telles choses. Tout ce que les autres expriment rejoint tellement ce que je me disais en mon for

${ }^{1}$ Les participantes portent des pseudonymes lorsqu'on présente le contenu des entrevues et des carnets. Les discussions de groupe peuvent se comparer à des groupes de réflexion, dans lesquels ceux qui prennent la parole sont habituellement non identifiables. Cependant, comme il y a eu aussi des discussions en petits groupes, les participantes à ces discussions ont été identifiées par leurs pseudonymes lorsque c'était possible. 
intérieur... Nous avons partagé beaucoup de choses; faire en sorte que cela ait lieu ainsi est vraiment significatif. C'est merveilleux.

Bridget a renchéri ainsi : «C'est réconfortant d'être avec des femmes qui ont vécu la même chose que moi... J'aime aussi sentir que je peux aider les autres. »

Le deuxième atelier a débuté avec une remarque de Barbara qui a commencé par reconnaître le soutien qu'elle avait reçu d'une autre participante : «J'ai pensé toute la semaine à votre commentaire sur mes écrits et je me suis dit : "Que c'est gentil!”. Alors je voulais vous en remercier. [S'adressant au groupe] Son commentaire m'a vraiment encouragée. » Cette remarque de Barbara a permis de recréer l'esprit rassurant du premier atelier dès le début du deuxième atelier.

Pendant l'atelier 2, Nathalie a aussi fait part de différentes manières de créer un endroit rassurant, tant par le partage du vécu que dans les écrits :

Ce que j'aime le plus des ateliers, c'est d'être en relation avec des femmes aux prises avec la même situation et qui peuvent comprendre ce que je traverse sans avoir à expliquer. Mais j'ai également acquis des habiletés pour l'écriture et j'ai appris des types d'écriture. Par exemple, je ne savais pas ce qu'était l'écriture libre, comment même commencer à écrire de la poésie. Alors même si je ne suis pas prête à creuser jusqu'au fin fond de mes émotions - chaque chose en son temps! - je retiens des choses pour plus tard et je sais que je pourrai les utiliser pour arriver à comprendre mon mental.

Les participantes ont commencé à écrire dans leur carnet dès le premier atelier et ont été invitées à continuer entre les deux rencontres. Malgré certaines hésitations initiales, les participantes ont senti que le carnet pouvait leur procurer un espace sécurisant. Karen a écrit : « Écrire fait ressortir des émotions que je n'avais pas exprimées. » Elle expliquait ne pas avoir exprimé ces émotions parce qu’elle voulait protéger sa famille de celles-ci. Elle ajoutait ceci : « Écrire mes émotions m'inquiète parce que ça laisse des traces... Mais je me suis donné la permission d'écrire certaines choses négatives. » Cette question d'intimité à protéger a été abordée au début de l'atelier, quand l'un des animateurs a donné plusieurs façons d'avoir un endroit sûr pour le carnet (le laisser aux animateurs entre les ateliers et écrire seulement dans le contexte du groupe, insister avec les membres de sa famille sur ce besoin de confidentialité, etc.).

Dans un même ordre d'idée, le carnet de Deborah vient illustrer l'espace sûr pour l'écriture. Elle a écrit qu'elle se sentait chanceuse d'avoir le soutien de nombreuses personnes dans sa vie, mais que ces personnes ne comprenaient pas toujours le combat qu'elle poursuivait pour sa survie :

J'ai trouvé difficile dernièrement de partager ce qui se passe à mon sujet et d'en discuter, tant sur les plans physique que psychologique... Il $y$ en a qui pensent que parce que je vais bien dans l'ensemble, c'est la fin de l'histoire. Le stress psychologique et émotionnel est pourtant significatif, mais certaines personnes ne le réalisent tout simplement pas.
Son carnet lui a offert un endroit où écrire les problèmes dont elle n'arrivait pas à discuter avec d'autres personnes.

En réfléchissant aux ateliers, Deborah a noté ceci dans son carnet : «Je suis contente d'avoir pris le temps d'y participer. Contente de ce que j'y ai appris. Contente d'avoir eu la possibilité de penser, d'écrire et de créer. Et, par-dessus tout, contente d'avoir pu échanger avec d'autres femmes. » Par conséquent, des endroits rassurants ont bel et bien été mis en place dans le cadre de l'atelier et de sa structure, ainsi que des activités de partage effectuées en groupe, et de l'analyse confidentielle associée aux travaux écrits individuels et de réflexion. Comme l'indiquent les mots de Karen et de Deborah, le thème de l'endroit rassurant est également lié au deuxième thème : se donner le droit, entre autres choses, d'écrire.

\section{Se donner le droit de penser à soi, retrouver son équilibre}

Écrire a permis aux participantes de noter les émotions qu'elles n'arrivaient pas à exprimer pendant les phases du diagnostic et du traitement. De nombreuses participantes ont également senti qu'avant d'écrire, elles devaient accepter de se donner du temps pour prendre du recul vis-à-vis les divers rôles qu'elles endossent dans leur vie. L'idée de « se donner le droit » était également liée au désir de retrouver un équilibre dans sa vie (entre ses besoins personnels, ceux des membres de sa famille et son travail, par exemple).

Plusieurs participantes ont dit qu'elles ne s'étaient permis d'avouer aucune émotion pendant l'établissement du diagnostic et le traitement. À titre d'exemple, Marie a dit qu'elle avait mis le processus émotionnel en attente :

"Je me suis dit "OK. Tu dois le faire. Ça va être difficile pendant un an, mais tu dois passer à travers" ». Ensuite je me suis ressaisie, je me suis prise en main et j'ai commencé à tout organiser comme si je n'allais pas être fonctionnelle pendant un an. J'ai donc fait un testament. J'ai demandé à mes amis et à ma famille d'aider mon mari avec les enfants. J'ai commencé à planifier les repas. J'ai poursuivi cette planification à un rythme effréné pendant les deux semaines qui ont précédé mon intervention chirurgicale afin que mon mari n'ait pas à le faire. J'ai payé les factures d'avance pour que nous ayons un crédit, parce que c'est moi qui moccupe des finances de la maison.

Marie a poursuivi :

J'avais décidé de tenir un journal sur un blogue et de tout raconter. Je pense que j'y ai écrit deux fois finalement et c'est tout! Puis j'ai eu de la chimiothérapie et de la radiothérapie et j'étais toujours centrée sur mon aspect physique. Maintenant que tout est terminé, je suis effondrée émotivement. Je pense que tout ceci me rattrape... Je ne me reconnais plus moi-même. Ça ne me serait jamais arrivé avant. Je suis toujours au bord des larmes. Je suis extrêmement sensible, voire même hypersensible. Je deviens très agressive. Je suis vraiment dans un état lamentable.

Une autre participante a exprimé des pensées similaires : « Juste d'avoir la liberté de pleurer m’a énormément aidée à libérer les émotions que j'avais enfouies au fond de moi... Pendant tout le traitement, je peux dire que je n'ai pas pleuré 
très souvent parce que j'ai essayé d'être forte. » Suzanne a notamment écrit ceci dans son carnet au sujet des phases du traitement : « Ne survenaient dans ma tête que l'horaire chaotique des rendez-vous chez les médecins et des examens, et tout l'inconnu de ce qui allait suivre. » Barbara a également mentionné ceci :

J'ai mis dix mois avant de pleurer au sujet de mon can-

cer. Ça m'a pris dix mois. J'ai fini par faire suffisamment confiance à quelqu'un. Je me suis assise et j'ai dit : "Je pensais que j'allais en mourir. " J'ai tellement pleuré, et c'était dans un endroit sécurisant. Puis en fin de compte, je me suis dit que j'avais finalement réussi à pleurer pour mon cancer... Il faut se donner la priorité, ce qui est vraiment difficile pour les femmes parce que nous donnons la priorité à beaucoup d'autres personnes avant nous.

Pour les participantes comme Barbara, le fait d'écrire et de participer aux ateliers a été associé à l'expression émotionnelle et à la possibilité de «se mettre au premier plan », de se donner le droit de le faire.

Cette notion de permission a également été reliée aux discussions sur l'équilibre entre soi, le travail et la famille. Nathalie a évoqué le sentiment que la fin du traitement avait simplement été suivie par un retour au « train-train bien occupé » d'avant le cancer.

Je suis passée à travers tous les traitements. J'ai subi une mastectomie et j'ai reçu de la chimiothérapie et de la radiothérapie, tout le nécessaire, et maintenant j'ai repris le travail à temps plein... Maintenant que je suis de retour au travail et que je redeviens aussi occupée qu'avant, j'ai l'impression que je dois trouver un exutoire. Je cours et c'est merveilleux, mais je dois trouver quelque chose d'autre pour gérer mon stress. Parce que je ne veux pas retomber dans la même vie occupée que j'avais avant. Pourtant, j'ai l'impression d'y être retombée. Alors, espérons que ceci m'aidera à gérer mon stress et à faire sortir tout ça.

De manière similaire, une autre participante a relaté ceci : J'adore écrire, mais je ne le fais jamais... Voici ce que j'en ai retiré : J'ai pensé à moi en premier et ça a été vraiment difficile pour moi parce que j'ai des enfants, des petits-enfants, et des responsabilités à mon boulot. Je fais passer tout le monde avant moi... Je réalise, en relisant ce que j'ai écrit, que j'ai besoin de trouver du temps. Je dois prendre le temps d'aller au chalet. J'ai besoin de contempler le lac. Je parle toujours au «je, je, je », bien entendu. Lorsque je fais cela, je me sens un peu coupable parce que je veux écrire «nous », mais c'est toujours «je, je, je » et puis je me dis « Ça me fait un bien énorme ». Parce que je trouve encore ça pénible d'être passée par là.

Lors de la discussion sur l'écriture, une autre participante a fait ressortir les relations entre l'atteinte de l'équilibre, le fait de donner priorité à ses propres besoins, et l'écriture :

Mon carnet a été une sorte de dialogue avec mon père... C'était comme s'il m'écrivait une histoire... Me rappelait ces sages paroles : «La vie est trop courte. Trouve du temps pour toi. Qu'est-ce que tu veux pour toi-même? Accorde-toi du temps pour faire ce que tu veux faire. »
D'autres participantes ont également écrit dans leur carnet de se donner le droit d'exprimer leurs émotions et de prendre soin d'elles avant tout. Suzanne, à ce sujet, a écrit : « Il est temps de réfléchir et de penser à moi. Les autres en seront-ils déçus? » Puis, plus loin dans ses écrits : «Je prends une pause. Je ralentis la cadence. J'établis mes priorités. Je réévalue. Je réfléchis. Je fais des choix... Je dois me recentrer sur moi et me concentrer sur ma guérison... C'est plus qu'une guérison physique, c'est aussi émotionnel et spirituel. » Pareillement, Barbara a noté ceci au sujet d'une approche large de la guérison : « Pour pouvoir sourire, rire et être en paix, j'ai besoin de soutien, de personnes agréables autour de moi, d'un milieu paisible... Je vais être égoïste en me protégeant des situations négatives. » De façon similaire, Nathalie a écrit : « J'ai besoin d'équilibre dans ma vie. D’un équilibre physique, mental, émotionnel et social. D’une liste de priorités. De juste dire non. Je dois mieux écouter mes envies, ce que je veux faire. Ce que je veux atteindre. C'est ça que je cherche. Puis-je y arriver? » Le cancer lui avait donné la possibilité de réévaluer ce qui était important, mais qu'après être retournée au travail pendant deux ans et demi, elle écrivait :

Je suis de nouveau happée par le stress de vouloir en faire trop en une journée. Je ne veux pas vivre ainsi. Je veux occuper mon temps intelligemment... Me donner la priorité... Je me suis engagée à m'accorder 15 minutes chaque jour juste pour moi. Pour faire une sieste, écrire, écouter de la musique, etc.

Bridget a écrit : «J'essaie de me débarrasser des “je devrais” dans ma vie. » Et Sandra : « Me permettre d'avoir du plaisir, et que du plaisir. Plus de "je pourrais, je devrais, il faudrait”. » Même s'il était reconnu comme précieux, il a semblé difficile de s'accorder le droit de prendre soin de soi. Marie a écrit au sujet d'une journée où elle se sentait fatiguée et incapable d'accomplir une liste de tâches qu'elle s'était dressée :

J'ai constamment l'impression que je ne fais pas ce que je serais censée faire. Faire le ménage. Ramasser. Planifier pour l'école. Apprendre à lire aux enfants. Dresser le chien. Préparer de bons repas santé. De l'exercice. Boire plus d'eau.

On remarque aussi chez les participantes que l'enseignement aux patients peut avoir des effets négatifs, comme le révèlent les écrits de Barbara : «Je suis obnubilée par la qualité de ma nourriture, la bonne façon de faire de l'activité physique. Je voudrais oublier tout cela pour entreprendre de nouvelles activités non associées au cancer. »

En résumé, les participantes ont indiqué qu'elles avaient l'impression de devoir demander la permission pour exprimer leurs émotions et que peu de possibilités de le faire durant le traitement s'étaient présentées. Lécriture et les discussions ont offert aux participantes cette possibilité d'exprimer leurs émotions et de prendre pour elles-mêmes du temps qu'elles auraient consacré à d'autres sinon. Vu les schémas hommefemme intégrés dans nos sociétés, ceci ne constitue pas une mince tâche, comme le démontrent ces femmes. De plus, même si prendre soin de soi était perçu de façon positive, cela provoquait quand même une pression pour se préparer à une éventuelle récidive, une source continuelle d'anxiété et d'incertitude. 


\section{Surmonter les craintes et combattre l'incertitude}

Les craintes et l'incertitude sont des facteurs temporels, comme l'indiquent les discussions des femmes et leurs carnets d'écriture. Ces notions sont mises en lien avec la cause du cancer et obligent la personne à revenir sur son passé. Les craintes et l'incertitude sont aussi reliées à la possibilité de récidive, toujours rappelée par les tests de suivi et l'attente des résultats. Ainsi, ces craintes se rattachent à la fois au présent et au futur. L'écriture a permis d'atténuer une partie de ces peurs.

La nature temporelle des craintes et de l'incertitude se reflète dans les écrits de Bridget sur la cause de son cancer : « Pourquoi ai-je eu le cancer? Qu'est-ce que j’ai mal fait? Mettre le doigt sur cette cause permettrait de mettre fin au comportement fautif, et d'éviter que ça se reproduise. » La réflexion se poursuivait sur trois pages, l'hormonothérapie et l'exposition aux pesticides étant notamment évoquées.

Suzanne illustre bien comment ce type d'anxiété était relié à son vécu actuel avec le traitement : «Peur, découragement, anxiété, incertitude, sentiment de perte, désespoir, solitude, tristesse, ne sont que quelques mots pour décrire mes sentiments lors des mauvais jours et lorsque le traitement est foudroyant... Personne ne sait ce dont demain sera fait, quel traitement empêchera le cancer de récidiver. » Marie a noté que, pendant le traitement, elle aurait apprécié qu'un professionnel de la santé lui dise que «ça irait ». Personne ne le lui a dit, et même si elle comprend qu'il est probablement « impossible » qu'un médecin dise cela, ces quelques mots auraient énormément diminué sa détresse alors qu'elle était au cœur même du traitement ou quand elle se remémorait cette période.

La peur s'est également reflétée dans les mots des participants au sujet de l'avenir. Une participante a ainsi déclaré :

Ma grande peur est de mourir... Je ne veux pas partir. Je ne veux pas mourir... Cette [image décrite dans le texte] transportait toutes ces connotations sur la famille et les gens que j'avais perdus, et ma crainte était que ce soit la fin pour moi aussi.

Karen a écrit : " C'est normal d'être effrayé; nous avons peur de l'inconnu. Dans ce cas, cet inconnu, ça pourrait être la mort. Le néant. Les personnes qu'on abandonne. Je suis effrayée. Je suis forte. » Ailleurs, elle écrivait dans son carnet : « Je suis effrayée. Je ne peux pas travailler. J'ai peur d'une récidive. J'ai peur d'avoir besoin d'autres traitements. J'ai peur des effets secondaires... Je ne sais pas comment dire aux gens ce qui m’arrive. » Également, Nathalie a écrit dans son carnet au sujet de l'incertitude et de la peur en utilisant une métaphore sur la « vie à Las Vegas ». Elle s'est demandé : «Qu'est-ce que fais avec ma peur d'une récidive? » Bridget a elle aussi noté ceci : « La plus grande chose à laquelle je dois faire face est la peur de la récidive. Je déteste même écrire à ce sujet parce que je ne veux pas y penser. Mais c'est là quand même. » Elle attendait les résultats de ses examens au moment où elle écrivait cela dans son carnet, tout comme Deborah, une autre participante : «Je pense que c'est ce que les survivants du cancer doivent vivre. C'est toujours là, dans un coin de notre tête. » Cependant, le dernier récit de Bridget décrivait une fontaine d'eau, qui devenait de plus en plus forte : «Un jet d'eau à la surface qui commence à faire des bulles et devient de plus en plus puissant... passant par toutes les couleurs de l'arcen-ciel. » Pour elle, l'image de l'eau représentait l'espoir; elle peut être «faible » ou «forte », mais toujours « là si on en a besoin».

Même si écrire procure un endroit rassurant et offre la possibilité d'exprimer ses craintes, les participantes ont également écrit au sujet des forces émergentes, comme le texte de Bridget au sujet de la fontaine le montre. Comme Bridget, Barbara a indiqué : «Je suis toujours en train de penser à l'avenir. Je peux participer et encore contribuer. Je peux apprendre et absorber l'information. Je ne suis plus paralysée par la peur. »

En résumé, les craintes et l'incertitude ont été associées au passé des participantes qui avaient terminé leur traitement, en grande partie dans le cadre des réflexions menées sur les choses ayant causé le cancer. Ces sentiments étaient également associés au présent chez les participantes encore sous traitement. Pour la plupart des participantes, les craintes et l'incertitude masquaient les considérations futures de la vie après le traitement contre le cancer, mais ont aussi suscité des écrits pleins d'espoir sur l'acceptation et ses forces personnelles.

\section{DISCUSSION}

Nos travaux de recherche confirment la quantité grandissante de données probantes en matière de besoins non comblés chez les survivants du cancer pendant la transition vers la survie (Boyes et al, 2012; Fitch et al, 2009; Thompson et al, 2014). Nos données renforcent l'idée selon laquelle la survie est un processus dynamique qui continue toute la vie (Pelusi, 2001), mais celles-ci soulignent également les lacunes relevées dans les soins, qui sont également signalées dans divers documents stratégiques sur la survie (Howell et al, 2009). Pour résoudre ces lacunes, les éducateurs pourraient commencer par tirer profit de la reconnaissance grandissante des approches d'art-thérapie en matière de survie, en élaborant, par exemple, des modules pour les professionnels de la santé. Malgré les durées variables entre le diagnostic et le traitement ( 6 mois à 9 ans), toutes les participantes ressentaient encore de la détresse, de l'incertitude et un manque de soutien psychosocial (par exemple, des endroits rassurants où les émotions pourraient être analysées et partagées). En ajoutant ces options à leur formation initiale, les infirmières seraient bien placées pour créer des endroits de ce genre.

Les participantes ont indiqué que vivre avec les difficultés liées à la survie, comme celles décrites ci-dessus, était la principale raison qui les avait poussées à s'inscrire aux ateliers et à écrire. Même si les participantes avaient l'occasion de définir les sources de détresse (par exemple, les aspects existentiels), il est difficile de savoir si cette détresse est vraiment bien saisie dans la littérature. Les futures recherches pourraient utiliser l'écriture et d'autres approches d'art-thérapie 
pour explorer qualitativement des dimensions plus complexes liées à la détresse auprès d'un échantillon plus vaste. Entre-temps, les programmes communautaires pourraient efficacement s'employer à résoudre des lacunes dans les programmes, et ce, à des coûts peu élevés, d'autant plus que les craintes et l'incertitude ne se terminent pas à la fin des soins actifs (Howell, Hack, Oliver, Chulak, Mayo, Aubin, Chasen et al, 2011; Thomas-MacLean, 2004a). La présence d'endroits rassurants pourrait procurer le soutien requis pour l'expression des émotions qui n'ont pas pu être analysées pendant les soins actifs ou qui peuvent surgir par la suite. Les administrateurs de programmes de survie voudront peut-être explorer des façons de créer et de maintenir ces endroits au moment de décider du lieu et des approches à privilégier pour répondre aux besoins non comblés de cette population dans le cadre de leur programme.

Nos résultats appuient également les diverses études qui indiquent que les interventions axées sur l'art-thérapie peuvent améliorer le bien-être des survivants du cancer (Lu et al, 2012; Luzzatto et Gabriel, 2000; Monti et al, 2006; Visser et Op'T Hoog, 2008). Toutes les patientes ont évalué le programme favorablement et ont indiqué qu'il répondait à de nombreuses préoccupations liées à la survie, comme la peur et la détresse. Les participantes recommandaient fortement d'offrir de nouveau cette intervention à d'autres personnes, si possible dans une formule plus longue, car elles auraient aimé avoir plus de temps pour écrire. Même si les bienfaits des arts pour la santé en général et de l'écriture en particulier sont de plus en plus reconnus, peu de programmes sont en place pour orienter les femmes qui souhaitent poursuivre ces pratiques de manière individuelle ou en groupe dans la communauté. Par exemple, la plupart des participantes qui avaient été conseillées par des professionnels de la santé mentale ou avaient participé à des groupes de soutien conventionnels s'étaient fait dire que l'écriture pourrait les aider pendant les phases de la survie. Cependant, aucune n'avait reçu de recommandations précises pour passer à l'action. À titre d'exemple, une participante avait acheté un carnet d'écriture quelque temps avant les ateliers, mais ne savait pas par où commencer ses écrits de réflexion. L'expérience des participantes révèle donc une lacune entre les travaux de recherche, les données probantes et la pratique. Afin de répondre à cette lacune, des programmes communautaires d'écriture pourraient servir d'intervention peu coûteuse, offrir les recommandations nécessaires et « donner le droit » aux participants de commencer à améliorer leur bien-être et leur qualité de vie. Jusqu'à ce que de tels programmes soient facilement accessibles, les professionnels de la santé auraient avantage à avoir sous la main une série de consignes d'écriture pour les remettre aux survivants du cancer et leur permettre ainsi d'acquérir des compétences de base en écriture si cette voie leur semble utile.

Comme souligné dans d'autres travaux de recherche (Mackenzie, 2014; Pelusi, 2001), plusieurs participantes ont également parlé des difficultés, dans le contexte des rôles familiaux à jouer et du travail rémunéré, à se faire passer en premier pour prendre soin d'eux-mêmes, que ce soit par des activités physiques ou de création, et ce, même si elles en connaissaient les bienfaits pour la santé. Arriver à équilibrer ces actes de soins et de compassion avec les exigences de la vie quotidienne s'est avéré difficile pour les participantes, ce qui met en évidence l'importance des interventions pour favoriser ce processus. Comme l'a fait remarquer une participante, le cancer lui a permis de comprendre l'importance de l'équilibre dans la vie. Cependant, elle a néanmoins été incapable de maintenir cet équilibre après son retour au travail. L'écriture et les discussions des participantes montrent que même si les activités de promotion de la santé réussissent à diffuser de l'information sur la prévention et la récidive du cancer, elles sont moins efficaces pour ce qui est de favoriser et de mettre en place des stratégies visant à prendre soin de soi. Nos données montrent que les messages visant à promouvoir la santé peuvent causer de la détresse lorsque l'engagement des utilisatrices ciblées est difficile ou que le contexte social (notamment les différences homme-femme normalisées par la société, par exemple en lien avec la prestation de soins) n'est pas considéré. Les femmes qui sont en phase de transition entre le traitement actif et la survie doivent se donner la permission de prendre du temps pour elles; les ateliers et l'écriture ont aidé à légitimer le temps passé à prendre soin de soi. En complément aux ateliers, ou dans le cas où il n'y en aurait pas, les professionnels de la santé pourraient rester attentifs aux différents rôles que les femmes remplissent et discuter des obstacles les empêchant de prendre soin d'elles lorsqu'ils donnent de l'information concernant la promotion de la santé.

Dans une large mesure, comparativement à l'écriture expressive de Pennebaker, qui se fait individuellement, nos données illustrent les bienfaits d'une approche de l'écriture effectuée en groupe. Toutes les participantes ont indiqué que le soutien du groupe ainsi que l'écriture guidée avaient été utiles. Les discussions de groupe ont contribué à la réflexion concernant le sens donné aux choses et ont aidé à recréer les récits en y trouvant les éléments positifs de la transition vers la survie. Les bienfaits des échanges n'ont pas seulement été notés dans les récits des participantes et dans les évaluations des ateliers, mais ont également été discutés au début du premier atelier, indiquant que les bienfaits de l'approche de groupe pourraient être quasi immédiats. Il est intéressant de faire remarquer que le cadre de l'étude prévoit un groupe de soutien traditionnel actif, mais que les participantes ont indiqué que même si elles voulaient discuter des problèmes liés à la survie, elles préféraient s'engager activement et apprendre tout en le faisant, signalant au passage que les groupes de soutien traditionnels n'offraient pas de telles possibilités. Cela pourrait signifier qu'il pourrait y avoir un lien bénéfique à explorer entre la science occupationnelle (par exemple l'activité occupationnelle) et les autres disciplines de la santé. Chercher à créer ces liens pourrait commencer dès l'étape des programmes éducatifs.

Malgré les bienfaits de l'écriture et les nombreuses retombées de nos travaux pour la pratique, les limites de notre étude doivent être reconnues. Premièrement, l'élaboration d'une étude longitudinale pourrait aider à faire la lumière sur 
certains des bienfaits et des obstacles associés à l'écriture, ce qui pourrait être raisonnablement perçu comme un exercice à long terme. Deuxièmement, les participantes étaient en majorité de race blanche, de classe moyenne et bien éduquées. Lécriture peut interpeller davantage ce type de population, et nos résultats peuvent avoir été influencés par l'autosélection des participantes, qui étaient donc forcément déjà prêtes à écrire. Cependant, bien que nous remarquions que certaines approches d'écriture (comme celle de l'Amherst Writers \& Artists) ont été utilisées avec succès dans des groupes marginalisés ayant un accès limité à l'éducation.

\section{RÉFÉRENCES}

Barone, T., \& Eisner, E.W. (2012). Arts based research. Thousand Oaks, CA: Sage Publications.

Boyes, A.W., Girgis, A., D'Este, C., \& Zucca, A.C. (2012). Prevalence and correlates of cancer survivors' supportive care needs 6 months after diagnosis: A population-based cross-sectional study. BMC Cancer, 12, 150. doi:10.1186/1471-2407-12-150

Bultz, B.D., \& Johansen, C. (2011). Screening for distress, the 6th vital sign: Where are we, and where are we going? Psycho-Oncology, 20(6), 569-571. doi:10.1002/pon.1986

Bury, M. (1982). Chronic illness as biographical disruption. Sociology of Health and Ilness, 4(2), 167-182. doi:10.1111/1467-9566.ep11339939

Camic, P.M. (2008). Playing in the mud: Health psychology, the arts and creative approaches to health care. Journal of Health Psychology, 13(2), 287-298. doi:10.1177/1359105307086698

Collie, K., Bottorff, J.L., \& Long, B.C. (2006). A narrative view of art therapy and art making by women with breast cancer. Journal of Health Psychology, 11(5), 761-775. doi:10.1177/1359105306066632

Cox, S.M., Lafrenière, D., Brett-MacLean, P., Collie, K., Cooley, N., Dunbrack, J., \& Frager, G. (2010). Tipping the iceberg? The state of arts and health in Canada. Arts \& Health, 2(2), 109-124. doi:10.1080 /17533015.2010.481291

Fitch, M., Ristovski-Slijepcevic, S., Scalzo, K., Bennie, F., Nicoll, I. et Doll, R. (2009). Survie au cancer : la création d'un programme national. Revue canadienne de soins infirmiers en oncologie, 19(2), 55-59.

Frank, A. (1995). The wounded storyteller: Body, illness, and ethics. Chicago, IL: University of Chicago Press.

Haltiwanger, E., Rojo, R., \& Funk, K. (2011). Living with cancer: Impact of expressive arts. Occupational Therapy in Mental Health, 27(1), 65-86.

Holland, J.C., \& Bultz, B.D. (2007). The NCCN guideline for distress management: A case for making distress the sixth vital sign. JNCCN Journal of the National Comprehensive Cancer Network, 5(1), 3-7.

Howell, D., Currie, S., Mayo, S., Jones, G., Boyle, M., Hack, T., ... Collacutt, V. (2009). A pan-Canadian clinical practice guideline: Assessment of psychosocial health care needs of the adult cancer patient. Toronto: Canadian Partnership Against Cancer (Cancer Journey Action Group) and the Canadian Association of Psychosocial Oncology.

Howell, D., Hack, T., Oliver, T., Chulak, T., Mayo, S., Aubin, M., \& Tompson, M. (2011). A pan-Canadian practice guideline: PanCanadian guidance on organization and structure of survivorship services and psychosocial-supportive care best practices for adult cancer survivors. Canadian Partnership Against Cancer (Cancer Journey Action Group) and the Canadian Association of Psychosocial Oncology: Toronto.
Malgré ces limites, nos travaux de recherche indiquent qu'une intervention d'écriture communautaire peut combler certaines lacunes présentes dans la recherche et la pratique en matière de survie au cancer. Offrir un endroit rassurant pour l'expression des émotions (peur, détresse...) tout en aidant les participantes à créer de nouveaux récits axés sur le bien-être. Les futures recherches pourraient porter sur la compréhension qualitative des bienfaits possibles à long terme qu'apporte la réflexion sur le sens des choses qui nous arrivent dans un contexte élargi de transition vers la survie.

Howell, D., Hack, T.F., Oliver, T.K., Chulak, T., Mayo, S., Aubin, M., ... Sinclair, S. (2011). Survivorship services for adult cancer populations: A pan-Canadian guideline. Current Oncology, 18(6), e265-281. doi:10.3747/co.v18i6.956

Jensen-Johansen, M., Christensen, S., Valdimarsdottir, H., Zakowski, S., Jensen, A.B., Bovbjerg, D., \& Zachariae, R. (2012). Effects of an expressive writing intervention on cancer-related distress in Danish breast cancer survivors-Results from a nationwide randomized clinical trial. Psycho-Oncology, 22(7), 1492-1500. doi:10.1002/pon.3193

Knowles, J.G., \& Cole, A.L. (2008). Handbook of the arts in qualitative research: Perspectives, methodologies, examples, and issues. Thousand Oaks, CA: Sage Publications.

Leininger, M. (1994). Evaluation criteria and critique of qualitative research studies. In J.M. Morse (Ed.), Critical issues in qualitative research methods (pp. 95-115). Thousand Oaks, CA: Sage Publications.

Lu, Q., Zheng, D., Young, L., Kagawa-Singer, M., \& Loh, A. (2012). A pilot study of expressive writing intervention among Chinesespeaking breast cancer survivors. Health Psychology, 31(5), 548. doi:10.1037/a0026834

Luzzatto, P., \& Gabriel, B. (2000). The creative journey: A model for short-term group art therapy with posttreatment cancer patients. Art Therapy, 17(4), 265-269. doi:1080/07421656.2000.10129764

Mackenzie, C.R. (2014). 'It is hard for mums to put themselves first': How mothers diagnosed with breast cancer manage the sociological boundaries between paid work, family and caring for the self. Social Science and Medicine, 117, 96-106. doi:10.1016/j. socscimed.2014.07.043

Malchiodi, C.A. (2013). Art therapy and health care. New York, NY: Guilford Press.

Merz, E.L., Fox, R.S., \& Malcarne, V.L. (2014). Expressive writing interventions in cancer patients: A systematic review. Health Psychology Review, 8(3), 339-361. doi:10.1080/17437199.2014.882007

Monti, D.A., Peterson, C., Kunkel, E.J., Hauck, W.W., Pequignot, E., Rhodes, L., \& Brainard, G.C. (2006). A randomized, controlled trial of mindfulness-based art therapy (MBAT) for women with cancer. Psycho-Oncology, 15(5), 363-373. doi:10.1002/pon.988

Munhall, P.L., \& Oiler-Boyd, C. (1993). Nursing research: A qualitative perspective. New York, NY: National League of Nursing.

Nicholls, S. (2009). Beyond expressive writing: Evolving models of developmental creative writing. Journal of Health Psychology, 14(2), 171-180. doi:10.1177/1359105308100201

Pelusi, J. (2001). The past sets the stage for the future: Follow-up issues facing long-term cancer survivors. Seminars in Oncology Nursing, 17(4), 263-267. doi:10.1053/sonu.2001.27921 
Puetz, T.W., Morley, C.A., \& Herring, M.P. (2013). Effects of creative arts therapies on psychological symptoms and quality of life in patients with cancer. JAMA International Medicine, 173(11), 960969. doi:10.1001/jamainternmed.2013.836

Rieger, K., \& Schultz, A.S. (2014). Exploring arts-based knowledge translation: Sharing research findings through performing the patterns, rehearsing the results, staging the synthesis. Worldviews on Evidence-Based Nursing, 11(2), 133-139. doi:10.1111/wvn.12031

Rosedale, M. (2009). Survivor loneliness of women following breast cancer. Oncology Nursing Forum, 36(2), 175-183. doi:10.1188/09. ONF.175-183

Sinding, C., \& Wiernikowski, J. (2008). Disruption foreclosed: Older women's cancer narratives. Health, 12(3), 389-411. doi:10.1177/1363459308090055

Sonke, J., Rollins, J., Brandman, R., \& Graham-Pole, J. (2009). The state of the arts in healthcare in the United States. Arts Q Health, 1(2), 107-135. doi:10.1080/17533010903031580

Statistiques canadiennes sur le cancer. (2015). Sujet particulier : Prévisions concernant le fardeau futur du cancer au Canada. Consulté sur http://publications.gc.ca/collections/collection_2016/statcan/ CS2-37-2015-fra.pdf

Stuckey, H.L., \& Nobel, J. (2010). The connection between art, healing, and public health: A review of current literature. American Journal of Public Health, 100(2), 254-263. doi:10.2105/AJPH.2008.156497
The Institute of Medicine and National Research Council. (2005). From cancer patient to cancer survivor: Lost in transition. National Academies Press.

Thomas-MacLean, R. (2004a). Memories of treatment: The immediacy of breast cancer. Qualitative Health Research, 14(5), 628-643. doi:10.1177/1049732304263658

Thomas-MacLean, R. (2004b). Understanding breast cancer stories via Frank's narrative types. Social Science and Medicine, 58(9), 16471657. doi:10.1016/S0277-9536(03)00372-1

Thomas-MacLean, R. (2005). Beyond dichotomies of health and illness: Life after breast cancer. Nursing Inquiry, 12(3), 200-209.

Thompson, C.A., Stan, D.L., Solberg Nes, L., Jenkins, S.M., Lackore, K.A., \& Pruthi, S. (2014). Breast cancer survivors' self-reported needs and preferences of survivorship care. Breast Journal, 20(1), 107-109. doi:10.1111/tbj.12221

Thorne, S. (2008). Interpretive description (Vol. 2). Walnut Creek, CA: Left Coast Press Inc.

Visser, A., \& Op'T Hoog, M. (2008). Education of creative art therapy to cancer patients: Evaluation and effects. Journal of Cancer Education, 23(2), 80-84.

Waller, A., Groff, S.L., Hagen, N., Bultz, B.D., \& Carlson, L.E. (2012). Characterizing distress, the 6th vital sign in an oncology pain clinic. Current Oncology, 19(2), e53-59. doi:10.3747/co.19.882 